\title{
A comparison of adeB gene expression levels under conditions of induced resistance by different drugs in vitro in Acinetobacter baumannii
}

\author{
WAN ZHU ${ }^{1}$, HUI WANG ${ }^{2}$ and JING-PING ZHANG $^{2}$ \\ ${ }^{1}$ Department of Nosocomial Infection Control; ${ }^{2}$ Division of Infectious Diseases, \\ The First Hospital of China Medical University, Shenyang, Liaoning 110001, P.R. China \\ Received September 6, 2015; Accepted January 6, 2016
}

DOI: $10.3892 /$ etm.2017.4242

\begin{abstract}
The present study was designed to analyze the difference of the adeB gene expression levels under conditions of induced resistance and to verify the significance of the AdeABC efflux pump in the in vitro induction of Acinetobacter baumannii (A. baumannii). Strains were isolated from blood or drainage samples in the First Hospital of China Medical University. The minimum inhibitory concentration (MIC) was determined by a broth microdilution method. In addition, an in vitro induction of drug-resistance tests was confirmed with the three drugs. The expression level of the adeB was determined by quantitative polymerase chain reaction (qPCR), and there were 19, 17 and 25 strains isolated from this test that were susceptible to amikacin, netilmicin and imipenem, respectively. Furthermore, 11, 15 and 8 stably resistant strains generated against amikacin, netilmicin and imipenem, respectively, by in vitro induction. The MIC values of all the imipenem-sensitive and imipenem-resistant strains decreased by no more than 2 -fold under carbonyl cyanide 3-chlorophenylhydrazone (CCCP)-containing conditions. The MIC values of 10 amikacin-resistant and 14 netilmicin-resistant strains decreased 4-fold or more under CCCP-containing conditions. Furthermore, qPCR revealed that none of the imipenem-sensitive or the imipenem-resistant strains expressed the adeB gene, but there were 10 amikacin-resistant and 5 netilmicin-resistant strains that expressed the adeB gene. In conclusion, the present study confirmed that in vitro induction of drug-resistance tests in sensitive A. baumannii strains could produce drug-resistance following long-term exposure to low doses of antibacterial drugs. In addition, amikacin
\end{abstract}

Correspondence to: Dr Jing-Ping Zhang, Division of Infectious Diseases, The First Hospital of China Medical University, 155 Nanjing Northern Street, Shenyang, Liaoning 110001, P.R. China E-mail: zjp809302@163.com

Key words: Acinetobacter baumannii, adeB, induce-resistance, carbonyl cyanide 3-chlorophenylhydrazone, quantitative polymerase chain reaction and netilmicin are more prone to producing drug-resistance compared to imipenem in vitro. The results of the efflux phenotype indicate the possible application of CCCP in the inhibition of the efflux system in some drugs. The inconsistency between efflux phenotype and qPCR of adeB indicates that other mechanisms may also be included in the induction of drug-resistance that work with the active mechanism in order to increase drug resistance to common clinically-used antimicrobial drugs.

\section{Introduction}

Acinetobacter baumannii (A. baumannii) are a type of gram-negative non-fermenting bacteria that widely exist in nature and on the surface of human skin. In the presence of a compromised immune system, A. baumannii may cause serious infections, including ventilator-associated pneumonia, sepsis, urinary tract infections and meningitis (1). Due to the use of invasive operation, broad-spectrum antibiotics and immunosuppressive agents in the last decade, A. baumannii has transformed from a monodrug-resistant to a multidrug-resistant or pandrug-resistant organism, and is now becoming the major pathogen of severe fatal nosocomial infections (2). Previous results have suggested that numerous mechanisms may lead to this resistance, and the active efflux mechanism is an important factor for mutidrug-resistance in A. baumannii (3). Since it is the unique efflux pump to A. baumannii, the AdeABC system is important in mediating drug-resistance, which belongs to the resistance-nodulation-division multidrug efflux system (4).

Therefore, the present study was designed to analyze the association between the differences in the expression level of the efflux pump adeB gene using an in vitro induction of a drug-resistance test in A. baumannii, and to verify the significance of the efflux mechanism in the induced drug-resistance among the three drugs, respectively. Therefore, research on the mechanisms underlying induced multidrug resistance is aimed at finding a solution to antimicrobial resistance.

\section{Materials and methods}

Bacterial strains. A. baumannii strains that were sensitive to amikacin $(n=19)$, netilmicin $(n=17)$ and imipenem $(n=25)$ were 
isolated from clinical blood or drainage samples in the First Affiliated Hospital of China Medical University (Shenyang, China) between January 2009 and December 2010. Strains were identified and initial antimicrobial susceptibilities were determined by the Vitek system (BioMérieux, Marcy-l'Étoile, France). All of the strains were non-repetitive, meaning that only one strain was collected from each patient.

Instruments and reagents. The instruments that were used for the experiments included a VITEK-2 automated microbial analyzer and Sakuma MIT-P bacterial multipoint inoculator (Sakuma Co., Ltd., Matsudo, Japan). A quantitative polymerase chain reaction (qPCR) kit was purchased from Takara Biotechnology Co., Ltd., Dalian, China. Reference standards of amikacin, netilmicin and imipenem were purchased from The European Pharmacopoeia (EP; Strasbourg, France). Luria-Bertani and Mueller-Hinton $(\mathrm{MH})$ broths and $\mathrm{MH}$ agar were purchased from Oxoid Ltd. (Nepean, ON, Canada). AdeB and 16S rRNA primers were synthesized by Sangon Biotech Co., Ltd. (Shanghai, China). RNA extraction kits were purchased from Thermo Fischer Scientific, Inc. (Waltham, MA, USA). Quantitative and reverse transcription PCR kits were purchased from GeneCopoeia, Inc. (Rockville, MD, USA), diethyl pyrophosphate from Tiangen Biotech Co., Ltd. (Beijing, China), and carbonyl cyanide 3-chlorophenylhydrazone (CCCP) and acetone from Sigma-Aldrich (Merck KGaA, Darmstadt, Germany).

Susceptibility testing. The minimum inhibitory concentration (MIC) values of all the strains to each drug were tested by the broth microdilution assay method, which was recommended by the Clinical and Laboratory Standards Institute (CLSI; 2012) (5). A 0.5 McFarland standard bacterial suspension was prepared, which was then diluted using MH broth (10-fold and twice) and were placed in the 96-well plates at different concentrations. The A. baumannii strains that were sensitive to amikacin $(n=19)$, netilmicin $(n=17)$ and imipenem $(n=25)$ were selected for this experiment according to the CLSI standards (2012) (5). Furthermore, Pseudomonas aeruginosa (P.aeruginosa) ATCC27853 and Escherichia coli ATCC29522 were both used as control strains.

Multi-step stable in vitro induction of drug-resistance. Selective medium containing an appropriate concentration of one antibiotic and $\mathrm{MH}$ broth was prepared in a 96-well plate. The initial concentration of the antibiotic was half of the MIC of the testing strains. The final concentration of bacterial suspension was $4-8 \times 10^{5}$ colony forming units $/ \mathrm{ml}$. In total a volume of $200 \mu \mathrm{l}$ was prepared in each well of the 96-well plate, and each well contained $100 \mu \mathrm{l}$ antimicrobial solution and $100 \mu \mathrm{l}$ bacterial suspension. The suspension was inoculated into the $\mathrm{MH}$ broth medium with ascending concentrations of antimicrobial drugs, until the drug concentration reached $128 \mu \mathrm{g} / \mathrm{ml}$. The $128 \mu \mathrm{g} / \mathrm{ml}$ was then selected as the final concentration for induction. The resistant strains were cultured another five times at this final concentration. Next, the surviving strains were transferred to the drug-free broth medium and inoculated twice to maintain resistant stability. Then the resistant strains were re-inoculated in the media with a drug concentration of $128 \mathrm{mg} / \mathrm{l}$. The surviving strains were considered to have stable drug-resistance. Finally, the stably resistant strains were inoculated on a blood plate to preserve the strains. When the strains grew poorly, the induction was repeated with the same or reduced concentration. If there was no growth for two generations (the same concentration) the strains were discarded. In addition, the drug-free and strain-free control broth wells and the culture in the blood plate were also established. Finally, the duration of induction for each drug was $\sim 4$ weeks.

Detection of an efflux phenotype. The 2-fold agar dilution method, recommended by the 2012 CLSI, was used for this experiment. The MIC values of amikacin, netilmicin and imipenem were detected. Meanwhile, the MH agar containing CCCP was prepared. CCCP was fully dissolved in $10 \mu \mathrm{g} / \mathrm{ml}$ acetone and the solution was passed through a sterile filter (0.22- $\mu \mathrm{m}$; Bioer, Hangzhou, China). The final concentration of CCCP was $10 \mu \mathrm{g} / \mathrm{ml}$ in which the strains could survive. The criteria for a positive efflux phenotype were defined as a MIC value with CCCP that decreased 4-fold or more compared to the MIC values without CCCP (6). Both the sensitive and resistant groups were used for the test, and $P$. aeruginosa ATCC27853 was selected for the quality control strain.

Detection of the expression level of efflux pump gene adeB by $q P C R$. Following bacterial RNA extraction, the total RNA was reverse transcribed into cDNA and RT-qPCR was performed. AdeB was the target gene and 16S rRNA served as an internal reference gene (4). The reaction was $20 \mu \mathrm{l}$ in total: $10 \mu 12 \mathrm{X}$ All-in-one qPCR mix, $2 \mu \mathrm{l}$ PCR forward and $2 \mu 1$ PCR reverse primers, $2 \mu 1$ cDNA templates, $0.4 \mu 150 \mathrm{X}$ Rox Reference Dye and $3.6 \mu \mathrm{l}$ purified water. The reaction conditions consisted of three phases: Predenaturation at $95^{\circ} \mathrm{C}$ for $10 \mathrm{~min}$; 40 cycles of denaturation at $95^{\circ} \mathrm{C}$ for $10 \mathrm{sec}$, annealing at $55^{\circ} \mathrm{C}$ for $20 \mathrm{sec}$, extension at $72^{\circ} \mathrm{C}$ for $15 \mathrm{sec}$; and one cycle of dissociation at $95^{\circ} \mathrm{C}$ for $10 \mathrm{sec}$. For both the target and the internal reference genes, $\mathrm{RT}$-qPCR reactions were conducted in two tubes simultaneously, and each reaction was repeated six times. The results were analyzed by the $\Delta \Delta \mathrm{Cq}$ method (7). One positive strain (for adeB) was selected for the control sample. Standardization was made by the subtraction of the mean $\mathrm{Cq}$ value of each adeB gene and the mean $\mathrm{Cq}$ value of the corresponding 16S rRNA (repeated three times for each sample), yielding a corrected $\Delta \mathrm{Cq}$ value. Following correction, subtraction of the $\Delta \mathrm{Cq}$ value of the resistant strain from the mean $\Delta \mathrm{Cq}$ value of the positive strain yielded $\Delta \Delta \mathrm{Cq}$. Finally, the relative mRNA expression level of the adeB gene in the resistant strain was calculated using the $2^{-\Delta \Delta C q}$ method.

\section{Results}

In vitro induction of drug-resistance. There were 19, 17 and 25 strains (sensitive to amikacin, netilmicin and imipenem, respectively) selected for in vitro induction under a single drug-condition. All the MICs of the 19 amikacin-sensitive strains were no more than $16 \mu \mathrm{g} / \mathrm{ml}, 17$ netilmicin-sensitive strains were no more than $8 \mu \mathrm{g} / \mathrm{ml}$ and for the 25 imipenem-sensitive strains were $\leq 4 \mu \mathrm{g} / \mathrm{ml}$. Following the in vitro induction test, 11, 15 and 8 resistant strains were generated with stable mutations, 
Table I. Detection of efflux phenotype of in vitro-induced isolates by three drugs.

\begin{tabular}{|c|c|c|c|c|c|c|}
\hline \multirow{2}{*}{$\begin{array}{l}\text { Strain } \\
\text { number }\end{array}$} & \multicolumn{2}{|c|}{$\operatorname{MIC}(\mu \mathrm{g} / \mathrm{ml})$} & \multicolumn{2}{|c|}{$\operatorname{MIC}(\mu \mathrm{g} / \mathrm{ml})$} & \multicolumn{2}{|c|}{$\operatorname{MIC}(\mu \mathrm{g} / \mathrm{ml})$} \\
\hline & Imipenem & $+\mathrm{CCCP}$ & Netilmicin & $+\mathrm{CCCP}$ & Amikacin & $+\mathrm{CCCP}$ \\
\hline 1 & 256 & 256 & 256 & 64 & 512 & 128 \\
\hline 2 & 256 & 128 & 256 & 64 & 512 & 128 \\
\hline 3 & 256 & 256 & 256 & 64 & 512 & 128 \\
\hline 4 & 256 & 256 & 256 & 256 & 512 & 128 \\
\hline 5 & 256 & 256 & 256 & 16 & 512 & 128 \\
\hline 6 & 256 & 256 & 256 & 32 & 512 & 128 \\
\hline 7 & 256 & 256 & 256 & 32 & 512 & 128 \\
\hline 8 & 256 & 256 & 256 & 32 & 512 & $<0.015$ \\
\hline 9 & & & 256 & 32 & 512 & 128 \\
\hline 10 & & & 256 & 64 & 512 & 128 \\
\hline 11 & & & 256 & 0.5 & 128 & 128 \\
\hline 12 & & & 256 & 64 & & \\
\hline 13 & & & 256 & 64 & & \\
\hline 14 & & & 256 & 64 & & \\
\hline 15 & & & 256 & 64 & & \\
\hline
\end{tabular}

MIC, minimum inhibitory concentration; CCCP, carbonyl cyanide 3-chlorophenylhydrazone.

respectively. Among them, the imipenem-resistant group had the longest induction time.

Result of the efflux phenotype. The MIC values of all the sensitive strains decreased less than 2-fold under CCCP-containing conditions for the three drugs, demonstrating the negative efflux phenotype. On the other hand, there were 10 strains resistant to amikacin showing decreased MIC values for 4-fold or more after addition of CCCP, 14 strains for netilmicin but none for imipenem. All the strains grew well on the $\mathrm{MH}$ plates containing only CCCP. Furthermore, decreased MIC values for 4-fold or more with CCCP showed a significant efflux pump phenotype. The details of the efflux pump phenotype are listed in Table I.

Results of the adeB gene expression levels by RT-qPCR. The amplification curves of the target and the internal reference genes were all presented as typical S-shape kinetic curves, with evident exponential and plateau phases. The corresponding melting curves only had a single peak, indicating good specificity. Furthermore, no adeB gene expression was observed in any of the initially susceptible strains or the imipenem-resistant group. The expression levels of all the internal reference 16S rRNA standards were good. Furthermore, there were 10 and 5 strains, respectively, for the amikacin- and netilmicin-resistant groups, as shown in Table II and Fig. 1. In addition, the electrophoretograms of the strains with positive expression of the efflux pump adeB gene are shown in Fig. 2, in which lanes 1-10 showed the results from the strains in the amikacin-resistant group and the last five lanes show the results from strains $3,5,11,13$ and 15 of the netilmicin-resistant group. These data indicated different copy number between the groups; specifically, the netilmicin-resistant groups had a higher copy number.

\section{Discussion}

A. baumannii has evolved from being mono- to multi-drug resistant or even into a pandrug-resistant organism. They have become a major pathogen involved in serious and fatal nosocomial infections, which is an important global issue (8). Previous studies show that the resistant mechanisms of A. baumannii include formation of inactivated enzymes, gene mutations in chromosomes, changes in outer membrane porins and the active drug efflux mechanism $(6,9,10)$. Among these, the active efflux mechanism is the main factor causing multi-drug resistance. Since the AdeABC-type active efflux system in A. baumannii BM4454 (3) was discovered by French researchers in 2001, a greater number of studies on the mechanisms of multi-drug resistance in A. baumannii have focused on active efflux. Although AdelJK, AdeDE, AdeFGH and other systems have been studied in recent years, the AdeABC-type active efflux system still remains unique and most closely associated to A. baumannii $(11,12)$.

As an inhibitor of the efflux systems, CCCP can freely diffuse across both sides of the lipid membrane. CCCP is a strong uncoupler that transmits protons to destroy the transmembrane electrochemical gradient, resulting in a loss of energy supply for transport proteins. CCCP can damage the active effects of the efflux system, increase drug accumulation in bacteria and recover the susceptibility of bacteria to drugs (13). A previous study on active efflux pump inhibitors by Chinese researchers has demonstrated that their reported inhibitory effects are different to the presently reported effects (6), but it is not clear how suitable CCCP is as an inhibitor for screening the AdeABC efflux pump in A. baumannii and the difference between different antibiotics. 
Table II. Detection of the expression levels of the active efflux pump gene adeB in the amikacin and netilmicin mutation groups by RT-qPCR.

\begin{tabular}{|c|c|c|c|c|c|}
\hline & $\mathrm{Cq}$ & $\mathrm{Cq}$ & $\Delta \mathrm{Cq}$ & $\Delta \Delta \mathrm{Cq}$ & \\
\hline Strains & adeB & 16S rRNA & Trial group & Trial group-control group & Copy number \\
\hline Amikacin 1 & 27.26 & 21.32 & 5.94 & -3.41 & 10.6294 \\
\hline Amikacin 2 & 19.09 & 14.74 & 4.35 & -5.00 & 32.0000 \\
\hline Amikacin 3 & 19.35 & 14.87 & 4.48 & -4.87 & 29.2426 \\
\hline Amikacin 4 & 24.46 & 20.66 & 3.80 & -5.55 & 46.8507 \\
\hline Amikacin 5 & 24.32 & 20.13 & 4.29 & -4.56 & 23.5883 \\
\hline Amikacin 6 & 22.36 & 17.02 & 5.34 & -4.61 & 24.4201 \\
\hline Amikacin 7 & 19.54 & 15.48 & 4.06 & -5.29 & 39.1244 \\
\hline Amikacin 8 & 25.42 & 21.87 & 3.55 & -5.80 & 55.7152 \\
\hline Amikacin 9 & 19.87 & 15.20 & 4.67 & -4.68 & 25.6342 \\
\hline Amikacin 10 & 23.87 & 19.29 & 4.58 & -4.77 & 27.2843 \\
\hline Netilmicin 3 & 19.61 & 15.75 & 4.86 & -4.49 & 22.4711 \\
\hline Netilmicin 5 & 19.50 & 17.16 & 2.34 & -7.01 & 128.2903 \\
\hline Netilmicin 11 & 21.82 & 17.82 & 4.00 & -5.35 & 40.7859 \\
\hline Netilmicin 13 & 20.16 & 15.86 & 4.30 & -5.05 & 33.1284 \\
\hline Netilmicin 15 & 20.81 & 17.46 & 3.35 & -6.00 & 64.0000 \\
\hline
\end{tabular}

Control group A was PRABA (Pandrug-resistant Acinetobacter baumannii), and the fluorescent reverse transcription-quantitative polymerase chain reaction $\mathrm{Cq}$ values of adeB and $16 \mathrm{~S}$ rRNA genes were 24.81 and $15.46, \Delta \mathrm{Cq}=9.35$, respectively.

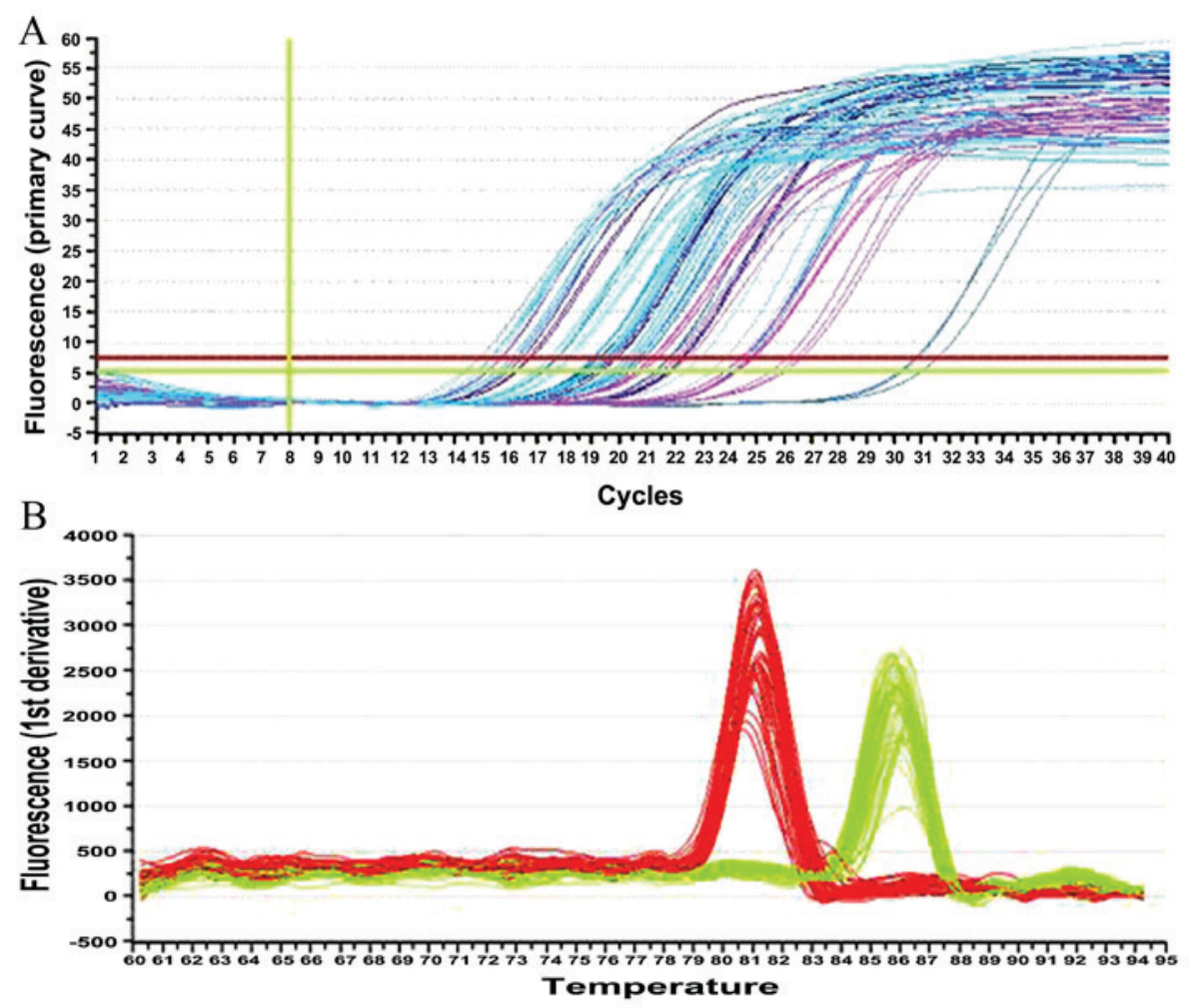

Figure 1. Relative real-time quantitation of adeB-positive strains (red: adeB; green: 16S rRNA). (A) Melting and (B) amplification curves.

The results of the present in vitro induction of drug-resistance tests demonstrate that amikacin and netilmicin are more prone to producing drug-resistance mutations than imipenem. Following induction by amikacin, netilmicin and imipenem in vitro, there were 10,14 and 0 resistant strains inhibited by CCCP, respectively. MIC values decreased 4 -fold or more after addition of the efflux pump inhibitor, CCCP. The most notable changes were in the amikacin- and netilmicin-resistant 

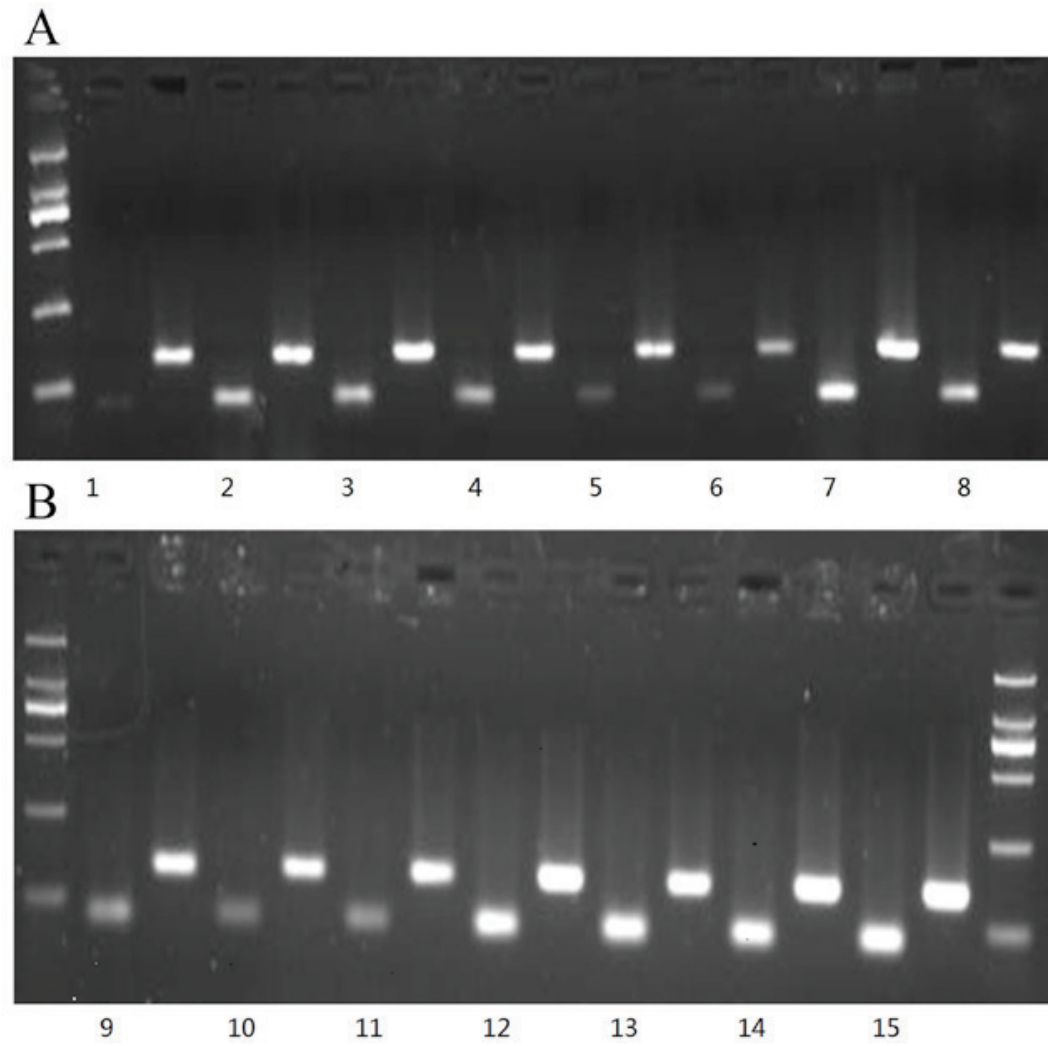

Figure 2. (A and B) Electrophoretograms of the efflux pump adeB positive-strains (odd lanes, adeB; even lanes, 16S rRNA).

groups with 11 and 15 breakpoints, respectively, indicating a marked efflux effect. These results demonstrate that amikacin and netilmicin are more likely to produce resistance caused by the AdeABC-type efflux system than imipenem in vitro. There were 15 strains that showed a positive efflux phenotype in netilmicin-resistant groups, but the expression of the adeB gene was detected only in 5 of all the 15 strains. In addition, the MIC value of strain no. 8 decreased 15 breakpoints when accompanied with CCCP, but its copy number was only 55.7152, as shown by RT-qPCR. Those results indicate the difference between different drugs in inducing resistance, and the application of CCCP for inhibition of efflux systems in different drugs.

The results demonstrated that among the drug-resistant strains that were induced in vitro, 10 and 5 strains expressed the adeB gene in the amikacin- and netilmicin-resistant groups, respectively. RT-qPCR fluorescence quantitative detection of resistant strains demonstrated that netilmicin-resistant strain no. 5 had a higher mRNA level of the adeB efflux pump and its copy number reached 128.2903. Furthermore, amikacin-resistant strain nos. 1, 5, 6, 9 and 10 and netilmicin-resistant strain no. 3 had lower mRNA levels of the adeB efflux pump. The copy number of amikacin-resistant strain no. 1 was 10.6294 , which was significantly lower than that of other strains. These results indicate that there are differences in the efficacy of common clinically used antimicrobial drugs due to the AdeABC efflux system in different strains. The induction time of netilmicin strain no. 5 was 13 days and the amikacin strain no. 1 was 19 days. Therefore, we can conclude that the induction time has no direct association with the expression of adeB. Furthermore, no expression of the adeB was detected by qPCR in initially susceptible strains, indicating that the active efflux mechanism is important in mediating the induction of drug resistance in A. baumannii (14-18). There were a number of differences between the adeB expression results and the number of in vitro induction of resistant strains. These findings indicate that drug-resistant strains possess efflux pump mechanisms or important resistance mechanisms other than the AdeABC efflux system, including extended-spectrum $\beta$-lactamases enzymes and changes in and loss of membrane permeability, all of which need to be studied further. By contrast to the reports on the mechanisms of carbapenem-resistance in A. baumannii, the resistance mechanisms have regional differences that may be caused by a different phenotype or genotype of the clinically collected strains (19-21).

The present study used an in vitro drug-induction test to confirm that susceptible A. baumannii could develop a drug-resistance following long-term exposure at low doses of antimicrobial drugs. In addition, the presence of an active efflux mechanism and the involvement of other mechanisms in drug resistance induction also increase the resistance against commonly used clinical antimicrobial drugs. Therefore, the rational use of antimicrobial drugs and strengthened management of treatment regimens can reduce or avoid formation of drug-resistant strains and evolution of resistance. Therefore, rational antibiotic use is an important risk management strategy used to prevent and reduce opportunistic pathogen infections in hospitals, and the development of drug-resistance.

\section{Acknowledgements}

The present study was funded by the Liaoning Province Natural Science Foundation (grant no. 2013021091). 


\section{References}

1. Bergogne-Bérézin E and Towner KJ: Acinetobacter spp. as nosocomial pathogens: Microbiological, clinical, and epidemiological features. Clin Microbiol Rev 9: 148-165, 1996.

2. Rodríguez Guardado A, Blanco A, Asensi V, Pérez F, Rial JC, Pintado V, Bustillo E, Lantero M, Tenza E, Alvarez M, et al: Multidrug-resistant Acinetobacter meningitis in neurosurgical patients with intraventricular catheters: Assessment of different treatments. J Antimicrob Chemother 61: 908-913, 2008.

3. Magnet S, Courvalin P and Lambert T: Resistance-nodulation-cell division-type efflux pump involved in aminoglycoside resistance in Acinetobacter baumannii strain BM4454. Antimicrob Agents Chemother 45: 3375-3380, 2001.

4. Peleg AY, Adams J and Paterson DL: Tigecycline efflux as a mechanism for nonsusceptibility in Acinetobacter baumannii. Antimicrob Agents Chemother 51: 2065-2069, 2007.

5. Clinical and Laboratory Standards Institute (CLSI): Performance Standards for Antimicrobial Susceptibility Testing: Twenty-First Informational Supplement. CLSI documen M100-S22. CLSI, Wayne, PA, 2012.

6. Shi WF, Jiang JP, Xu N, Huang ZM and Wang YY: Inhibitory effects of reserpine and carbonyl cyanide $\mathrm{m}$-chloro-phenylhydrazone on fluoroquinolone resistance of Acinetobacter baumannii. Chin Med J (Engl) 118: 340-343, 2005.

7. Livak KJ and Schmittgen TD: Analysis of relative gene expression data using real-time quantitative PCR and the 2(-Delta Delta C(T)) Method. Methods 25: 402-408, 2001.

8. Giannouli M, Cuccurullo S, Crivaro V, Di Popolo A, Bernardo M, Tomasone F, Amato G, Brisse S, Triassi M, Utili R and Zarrilli R: Molecular epidemiology of multidrug-resistant Acinetobacter baumannii in a tertiary care hospital in Naples, Italy, shows the emergence of a novel epidemic clone. J Clin Microbiol 48: 1223-1230, 2010.

9. Fernandez-Cuenca F, Martínez-Martínez L, Conejo MC, Ayala JA, Perea EJ and Pascual A: Relationship between beta-lactamase production, outer membrane protein and penicillin-binding protein profiles on the activity of carbapenems against clinical isolates of Acinetobacter baumannii. J Antimicrob Chemother 51: 565-574, 2003.

10. Lee JK, Martínez-Martínez L, Conejo MC, Ayala JA, Perea EJ and Pascual A: Mutations in the gyrA and gyrC genes in ciprofloxacin-resistant clinical isolates of Acinetobacter baumannii in Korea. Microbiol Immunol 49: 647-653, 2005.

11. Yoon EJ, Courvalin P and Grillot-Courvalin C: RND-type efflux pumps in multidrug-resistant clinical isolates of Acinetobacter baumannii: Major role for AdeABC overexpression and AdeRS mutations. Antimicrob Agents Chemother 57: 2989-2995, 2013.
12. Rajamohan G, Srinivasan VB and Gebreyes WA: Molecular and functional characterization of a novel efflux pump, AmvA, mediating antimicrobial and disinfectant resistance in Acinetobacter baumannii. J Antimicrob Chemother 65: 1919-1925, 2010.

13. Ni W, Li Y, Guan J, Zhao J, Cui J, Wang R and Liu Y: Effects of efflux pump inhibitors on colistin resistance in multidrug-resistant gram-negative bacteria. Antimicrob Agents Chemother 60: 3215-3218, 2016

14. Principe L, D'Arezzo S, Capone A, Petrosillo N and Visca P: In vitro activity of tigecycline in combination with various antimicrobials against multidrug resistant Acinetobacter baumannii. Ann Clin Microbiol Antimicrob 8: 18, 2009.

15. Ruzin A, Keeney D and Bradford PA: AdeABC multidrug efflux pump is associated with decreased susceptibility to tigecycline in Acinetobacter calcoaceticus-Acinetobacter baumannii complex. J Antimicrob Chemother 59: 1001-1004, 2007.

16. Lin L, Ling BD and Li XZ: Distribution of the multidrug efflux pump genes, adeABC, adeDE and adeIJK, and class 1 integron genes in multiple-antimicrobial-resistant clinical isolates of Acinetobacter baumannii-Acinetobacter calcoaceticus complex. Int J Antimicrob Agents 33: 27-32, 2009.

17. Poole K: Outer membranes and efflux: The path to multidrug resistance in Gram-negative bacteria. Curr Pharm Biotechnol 3: 77-98, 2002.

18. Zhang JP, Zhu W, Tian SF, Chu YZ and Chen BY: Molecular characteristics and resistant mechanisms of imipenem-resistant Acinetobacter baumannii isolates in Shenyang, China. J Microbiol 48: 689-694, 2010.

19. Heritier C, Poirel L, Lambert T and Nordmann P: Contribution of acquired carbapenem-hydrolyzing oxacillinases to carbapenem resistance in Acinetobacter baumannii. Antimicrob Agents Chemother 49: 3198-3202, 2005.

20. Hu WS, Yao SM, Fung CP, Hsieh YP, Liu CP and Lin JF: An OXA-66/OXA-51-like carbapenemase and possibly an efflux pump are associated with resistance to imipenem in Acinetobacter baumannii. Antimicrob Agents Chemother 51: 3844-3852, 2007.

21. Rhomberg PR and Jones RN; MYSTIC Program (USA) Study Group: Antimicrobial spectrum of activity for meropenem and nine broad spectrum antimicrobials: Report from the MYSTIC program (2002) in North America. Diagn Microbiol Infect Dis 47: 365-372, 2003. 